\title{
The Effect of Complementary Stereotypes on Impression Formation: A study of the rich and poor
}

\author{
$\mathrm{Su} \mathrm{Tao}^{1}$, Yan $\mathrm{Xu}^{2}$, and Cancan Yuan ${ }^{1}$ \\ 1) School of Ideological and Political Education, University of Geosciences (Beijing), Beijing, China (tao_su@126.com) \\ 2) School of Psychology, Beijing Normal University, Beijing, China
}

\begin{abstract}
To investigate how the stereotypes of the rich and poor affect impression formation, a situational simulation questionnaire is conducted to 192 participants. The experiment is a univariate between-participant design. The independent variable is social group of the target person (rich vs. poor). The dependent variables are rating valence of target person on competence, warmth and moral dimensions. Results show that, by the same neutral behavior, the rich target person has been seen as high competence, mid warmth but low moral; the poor target person has been seen as low competence, mid warmth and mid moral. There is a negative correlation between competence, warmth and moral dimensions. Conclusion: The stereotypes of the rich and poor present complementary characteristics, which bias the impression formation process and affect the explanation and attitude on relevant social events.
\end{abstract}

Keywords - complementary stereotypes, competence, warmth, moral

\section{补偿性刻板印象对印象形成的影响:以贫富群体为例}

\author{
陶塑 ${ }^{1}$ 许燕 $^{2}$ 袁灿灿 $^{1}$ \\ 1) 中国地质大学 (北京) 思想政治教育学院应用心理学研究所, 北京, 中国 \\ 2) 北京师范大学心理学院, 北京, 中国
}

摘 要 为了探讨对贫、富群体成员的刻板印象如何影响民众的印象形成过程, 本研究采用情境问卷法, 对 192 名被试进行实验研 究。结果表明: 对于同样的中性行为事件, 富人被认为是高能力、中等热情、低道德的, 穷人被认为是低能力、中等热情、中等道德 的; 被试对目标人物在道德维度上的评价与在能力、热情维度上的评价呈负相关。这说明, 民众对贫、富群体的刻板印象在不同维度 上呈现出补偿性特征，这种会使民众对群体成员的印象形成出现偏差，进而影响对相关社会事件的解释和态度。

关键词 刻板印象补偿, 能力, 热情, 道德

\section{1. 引言}

近年来, 涉及到优势、弱势群体成员的热点事件频发, 很多民众一提起官员、富人、“富二代”等优势群体, 就会 迅速产生冷漠、道德感缺失等消极印象; 而对于穷人、小 商贩等弱势群体则给予了更多同情, 认为他们是善良的、 是社会问题的受害者。这些都是由群体所处社会地位差异 带来的刻板印象, 将导致民众从明显带有偏向性的视角去 分析和判断事件。

在社会性偏向研究中, 刻板印象 (stereotype)、偏见

北京市哲学社会科学规划项目支持 (项目号: 12SHC024);

中央高校基本科研业务费专项资金支持（项目号：2-9-2012-123） （prejudice）和歧视（discrimination）是三个紧密关联的领 域, 刻板印象属于一种社会认知偏差, 偏见是以刻板印象 为基础的、对目标人物或群体的情感反应偏向, 而歧视则 是基于刻板印象和偏见的行为偏向 ${ }^{[1]}$ 。所以, 刻板印象自然 就成为理解社会性偏向的一个基本问题。

Fisk 等人提出的刻板印象内容模型 (Stereotype Content Model, SCM) 通过群体之间在社会结构上的相互关系, 预 测他们的刻板印象内容 ${ }^{[2]}$ 。该理论是以热情 (Warmth) 和 能力 (Competence) 为基础建立的二维模型, 刻板印象的 内容是在热情和能力两个维度上的评价的组合。研究发现, 很多群体的刻板印象是混合的、矛盾的, 有对某一个维度 的积极评价和另一个维度的消极评价组成, 竞争力弱的人 
被认为是热情的, 而竞争力强的人则不是; 地位高的人被 认为是高能力的, 而社会地位低的人则不是。这些关系在 对实际群体 ${ }^{[3]}$ 、实验建构的群体 ${ }^{[4]}$ 和对个体的知觉 ${ }^{[5]}$ 的研究 中都得到了验证。

SCM 模型虽在全球 17 个国家和地区得到了基本的验 证, 具有很好的文化普适性和群际关系预测性 ${ }^{[6]}$, 但中国研 究者发现, 民众对贫、富群体的刻板印象内容除能力和热 情外, 道德也是重要的衡量维度, 且混合性特征更多体现 在能力和道德维度。民众赋予贫、富群体各自补偿性的特 质, 即认为两者各自拥有独特的、相互抵消的优点和缺点, 富人被认为是高能力低道德的, 穷人被认为是低能力高道 德的 ${ }^{[7]}$ 。

本研究以能力、热情、道德三维度模型为基础, 采用 情境问卷的方法, 探讨刻板印象如何影响民众对贫、富群 体成员的印象形成, 即考察凸显了个体所属群体后, 被试 对该个体在人格特点上的描述和评价是否有差异, 及这种 差异是否呈现出补偿性特征。

\section{2. 研究方法}

2.1 被试

192 人, 年龄范围为 $18 \sim 33$ 岁, 平均年龄 $22.37 \pm 2.418$ 岁。被试被随机分配到阅读贫、富文本的两组。

\section{2 研究工具}

采用 Echterhoff 等人的实验范式 ${ }^{[8]}$, 选取的在能力、热 情、道德维度上, 能够代表贫、富群体突出特点的若干形 容词, 自编模棱两可的描述人物事件的文章。文章描述了 目标人物的若干种行为事件, 这些行为事件都是中性的, 不包含任何评价性语言, 被试根据事件推论出目标人物积 极或消极特质的可能性相同。这些行为事件也不具有群体 代表性, 既适用于富人, 也适用于穷人。文章样例如下 (括 号中的内容为可能推断出的特质):

XXX 做事情时并不会考虑太久，也很少询问别人的意 见。他会按照自己的想法, 迅速做出重要的决定。（果断一 草率)

当有人与 XXX 意见不一致时, 他很少反验。他说: “每 个人都有自己的观点, 别人改变不了我的想法, 我也不想 改变别人”。(随和一被动)

上学时, XXX 会向老师揭发那些违反学校纪律, 比如 考试作弊、上课说话的同学。他曾对自己的朋友说过：“我 有自己的原则, 我上学时从来都没违反过纪律, 也从来不 说谎。”(道德—虚伪)
共编制了 11 件行为事件, 分别属于能力、热情、道德 三个维度。事件编制完成后, 由 25 名心理学专业的硕博士 研究生, 对每个事件表现出的特质及效价进行评定。剔除 效价过高或过低的文本, 修改后最终保留 6 个行为事件, 每个维度 2 个, 形成一篇含 6 种特质的、中性的、对目标 人物行为事件描述的文章。

\section{3 研究程序}

告知被试, 本实验的目的是为了研究“对陌生人的印象 形成机制”的, 而被试的任务是根据阅读到的某个人的行为 对他进行评价, 并形成整体印象。先通过一段文字, 对目 标人物所属群体进行操纵。一半的被试读到的目标人物身 份是富人, 一半被试读到的目标人物身份是穷人。随后, 被试阅读描述目标人物行为的文章, 并根据原始文章, 为 每种行为特点写一个形容词, 描述该事件反映了何某怎样 的性格特点。通过对形容词维度、效价的分析, 可以反映 出被试将目标人物所属群体的刻板印象投射到印象形成和 事件解释中。

实验结束后, 给予报酬。

\section{4 研究程序}

本研究为 2 因素 (目标人物所属社会群体: 贫 vs.富) 被试间设计; 因变量为在能力、热情、道德维度上的描述 效价及整体效价。

\section{3. 研究结果}

\section{1 操纵检验}

为了检验实验操纵是否成功, 询问被试是否感知到了 目标人物的社会经济地位状况: “你觉得何某的社会经济地 位如何？”，1（贫穷） 7（富有）点评定。剔除感知目标 人物经济地位错误的被试 8 人, 剔除自身社会经济地位极 端的被试 2 人, 剩余 182 名被试。被试对富有目标人物的 社会经济地位感知平均值为 $6.11 \pm 0.774$, 对贫困目标人物 的社会经济地位感知平均值为 $2.42 \pm 0.873$, 差异显著 $(t=26.520, d f=138, p<0.001)$, 即对目标人物所属群体操 纵成功。

\section{2 评价效价的描述统计分析}

由两名心理学专业的研究生对被试列出的形容词分别 单独进行编码, 并对其积极和消极程度进行 -3 (极消极) +3 (极积极) 的 7 点评分, 评分者一致性 $r=0.96$, 将两位 评分者评定的效价求平均, 作为每个词效价的得分。将每 个被试在不同维度对贫富群体进行描述的形容词效价分别 
进行加合, 得到其在各维度上对贫富群体的评价, 即能力 效价、热情效价、道德效价; 将每个被试对贫富群体进行 描述的形容词效价全部加合, 得到其对贫富群体评价的整 体效价。对贫富群体的各维度效价和整体效价的描述统计 分析, 及以目标人物所属群体为自变量进行独立样本 $t$ 检验 的结果见表 1 。

从表中可以看出, 被试对富有目标人物的整体评价更 为积极, 对贫困目标人物的整体评价略显消极。对于同样 的事件, 富人被认为是高能力的, 穷人被认为是低能力的; 富人被认为是高热情的, 而对穷人的热情评价更接近中性; 富人被认为是低道德的, 而对穷人的道德评价也更接近中 性。

表1 对贫、富群体描述的效价分析表

\begin{tabular}{|c|c|c|c|c|}
\hline 维度 & 群体 & $\mathrm{N}$ & $\mathrm{M} \pm \mathrm{SD}$ & $\mathrm{t}$ \\
\hline \multirow{2}{*}{ 能力 } & 富 & 93 & $0.28 \pm 0.39$ & \multirow{2}{*}{$7.501 * * *$} \\
\cline { 2 - 4 } & 贫 & 89 & $-0.20 \pm 0.46$ & \\
\hline \multirow{2}{*}{ 热情 } & 富 & 93 & $0.18 \pm 0.30$ & \multirow{2}{*}{$2.948^{* *}$} \\
\cline { 2 - 4 } & 贫 & 89 & $0.04 \pm 0.34$ & \\
\hline \multirow{2}{*}{ 道德 } & 富 & 93 & $-0.16 \pm 0.43$ & \multirow{2}{*}{$-1.913^{*}$} \\
\cline { 2 - 4 } & 贫 & 89 & $-0.04 \pm 0.43$ & \\
\hline \multirow{2}{*}{ 整体效价 } & 富 & 93 & $0.10 \pm 0.20$ & \multirow{2}{*}{$5.294 * * *$} \\
\cline { 2 - 4 } & 贫 & 89 & $-0.06 \pm 0.22$ & \\
\hline
\end{tabular}

注: *表示在.05 水平上显著, **代表在.01 水平上显著。

\section{3 刻板印象补偿效应分析}

计算被试对各维度评定效价间的相关, 发现 $\mathrm{r}$ 能力_热情

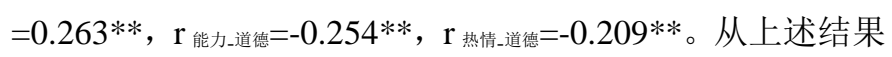
可以看出, 被试对目标人物在道德维度上的评价与在能力、 热情维度上的评价呈负相关, 即出现了刻板印象补偿效应, 对目标人物的能力和热情评价越积极, 对其道德的评价越 消极。

\section{4. 讨论}

本研究自编了情境文本, 控制事件发生主体, 对补偿 性刻板印象对印象形成的影响进行了测量。研究编制了关 于某个目标人物的若干模棱两可的行为事件, 所有这些行 为事件本身都是中性的。被试对这些行为事件的评价受目 标人物所属群体的影响, 会把对群体的刻板印象和态度投 射到目标人物身上, 从而对其行为做出不同的评价和解释。 Echterhoff 等人的研究结果发现, 对于同样的行为事件, 被 试对内群体目标人物 (同校的学生) 的描述和评价比对外 群体目标人物 (职高生) 更为积极 ${ }^{[8]}$ 。在本研究中, 也出现
了差异性结果, 被试对贫、富目标人物在各维度上的描述 效价均差异显著: 对富人的整体描述比对穷人更积极; 在 能力和道德维度上, 富人的行为被解释为高能力、低道德 的, 穷人的行为被解释为低能力、高道德的; 而在热情维 度上, 被试对富人和穷人的热情描述都处于中等水平。各 维度间相关表明, 对贫、富群体行为的解释在道德维度和 能力维度上存在负相关, 即出现了道德对能力的补偿。而 且, 本研究结果与刻板印象内容模型不同, 被试对贫、富 群体的刻板印象中, 热情并非补偿的重要和稳定维度。

刻板印象作为一种特定的社会认知图式, 对人们的社 会认知和行为有着重要的指导性作用。出于节省认知资源、 促进认知加工的目的, 人们会倾向于采取认知捷径, 根据 对方所属的社会类别直接决定自己的应对方式 ${ }^{[9]}$ 。首先, 刻 板印象影响个体对信息的注意及选择过程, 能够帮助个体 在纷繁复杂的信息中迅速捕捉到有价值的信息, 当认知资 源有限时, 刻板印象起到过滤器的作用, 使个体有选择地 注意那些与刻板印象一致的信息, 而不一致的信息会被作 为偶然或意外被忽略。其次, 刻板印象影响个体对信息的 编码和加工, 与刻板印象有关 (一致或不一致) 的信息存 在加工优势, 进而产生相应的记忆。这解释了民众对贫富 群体的刻板印象内容是如何影响到对相关群体成员的印象 形成的。由于对事件当事人的偏向性印象会影响个体对事 件的归因, 当外部信息不完整时, 人们会据此对行为和事 件做出解释, 即对社会热点事件持有偏向性的态度和判断。

这些偏向性的根源来自对优势、弱势群体的刻板印象, 尤其是对优势群体道德方面的消极评价, 民众难以将对事 件的判断和对群体的刻板印象分离出来。要改变这一现状, 首先要避免给当事人“贴标签”, 以减少刻板印象的激活; 同时营造良好、宽容的社会氛围, 促进群体间的融合, 尽 量消除对立刻板印象的存在。

\section{参考文献(References)}

[1] Fiske, S. T, Social Beings: A core motives approach to social psychology. John Wiley \& Sons, 2004.

[2] Fiske, S. T., Cuddy, A. J. C., Glick, P. S., \& Xu, J., A model of (often mixed) stereotype content: Competence and warmth respectively follow from perceived status and competition. Journal of Personality and Social psychology, 82, 878-902, 2002.

[3] Cuddy, A. J. C., Fiske S. T., \& Glick, P., The BIAS Map: Behaviors from intergroup affect and stereotypes. Journal of Personality and Social Psychology, 92, 631-648, 2007.

[4] Oldmeadow, J., \& Fiske, S. T., Ideology moderates status=competence stereotypes: Roles for belief in a just world and social dominance orientation. European Journal of Social 
Psychology, 37, 1135-1148, 2007.

[5] Russell A M T, Fiske S T. It's all relative: Competition and status drive interpersonal perception. European Journal of Social Psychology, 38(7): 1193-1201, 2008.

[6] Cuddy, A. J. C., Fiske, S. T., \& Kwan V. S. Y. et al., Stereotype content model across cultures: Towards universal similarities and some differences. British Journal of Social Psychology, 48, 1-33, 2009.

[7] Tao Su. The complementary stereotypes of rich and poor: effects of personal and system justice belief. Doctoral dissertation of Beijing Normal University, 2010.

[8] Echterhoff G, Higgins E T, Groll S., Audience-tuning effects on memory: the role of shared reality. Journal of personality and social psychology, 89, 257-276, 2005.

[9] Du Xiufang, New progress on the stereotype explorations. Journal of Hebei Normal University Educational Science Edition, 6(6): 105-108, 2004. 